\title{
The Development and Application of a Resilience-Promoting Program Using Changes in Sleep-Related Habits in Korean Adolescents
}

\author{
Hyoseung Kang 1,2, So-Jin Lee ${ }^{2,3}$, Bong-Jo Kim²,3, Cheol-Soon Lee ${ }^{2,4}$, Boseok Cha ${ }^{2,3}$, Dongyun Lee ${ }^{2,4}$, \\ Jiyeong Seo ${ }^{4}$, Jae-Won Choir, ${ }^{2,3}$, Young-Ji Lee ${ }^{4}$, Yoon Jung Lee ${ }^{3}$, and Eun-Ji Lim ${ }^{4}$ \\ ${ }^{1}$ Hanbit Psychiatric Clinic, Jinju, Korea \\ ${ }^{2}$ Department of Psychiatry, Gyeongsang National University School of Medicine, Jinju, Korea \\ ${ }^{3}$ Department of Psychiatry, Gyeongsang National University Hospital, Jinju, Korea \\ ${ }^{4}$ Department of Psychiatry, Gyeongsang National University Changwon Hospital, Changwon, Korea
}

\begin{abstract}
Objective: Resilience-promoting program which covers strategies to improve sleep health through modulation of sleep-related habits has been lacking. The authors aimed to develop a resilience-promoting program that incorporates the encouragement of healthy sleep habits. Methods: Fifty-eight adolescents (27 female, 46.6\%; aged $13.78 \pm 0.82$ years) were recruited to test the effectiveness of the program. During the first and final sessions, several self-reported questionnaires, including the Connor-Davidson Resilience Scale (CD-RISC), the Smartphone Addiction Scale-short version (SAS-SV), Hospital Anxiety and Depression Scale (HADS), Composite Scale of Morningness (CSM), Insomnia Severity Index (ISI), and a sleep habit-related questionnaires were administered to the adolescents. After the first session, adolescents were provided with an Actiwatch and were asked to wear the watch to the last session date. Multiple linear regression analyses were used to identify predictors of resilience and sleep quality. Results: The participants' resilience, morningness, sleep quality, wake after sleep onset (WASO), and wakening frequency during the night changed significantly. Resilience at the last session was related to sleep quality $(\beta=-0.394$, $\mathrm{p}=0.005$ ) at the last session when controlling for age, sex, and morningness. Sleep quality at the last session was related to the mean sunlight exposure time $(\beta=-0.363, p=0.037)$ at the last session when controlling for age, sex, and WASO during the third week. Conclusion: This study found that four weekly sessions of resilience-promoting program improved resilience, sleep quality including objective actigraphic measures, and morningness. Improved resilience was related to better sleep quality.
\end{abstract}

Keywords: Resilience-promoting program; Psychological resilience; Sleep quality; Smartphone addiction; Morningness; Actigraphy

Received: August 9, 2021 Revised: September 4, 2021 Accepted: September 4, 2021

Corresponding author: So-Jin Lee, MD, PhD, Department of Psychiatry, Gyeongsang National University School of Medicine, Gyeongsang National University Hospital, 79 Gangnam-ro, Jinju 52727, Korea.

Tel: 82-55-750-8086, Fax: 82-55-759-0003, E-mail: lifeofzoe@gnu.ac.kr

(a) This is an Open Access article distributed under the terms of the Creative Commons Attribution Non-Commercial License (https://creativecommons.org/licenses/bync/4.0) which permits unrestricted non-commercial use, distribution, and reproduction in any medium, provided the original work is properly cited.

\section{INTRODUCTION}

Resilience can be considered as the ability to adapt to various work and social situations, as well as to different states of physical and mental health [1]. Promotion of resilience is important to prevent various mental health problems [2]. Strategies for promoting resilience include cognitive, behavioral, and physical health interventions, emotion regulation, and social skills training, as well as modulation of neurobiological factors (especially through psychiatric intervention) [2]. Social skills may be influenced by an individual's psychological development, as well as the social environment and networks [2]. Promotion of physical health, emotional regulation, and cognitive and behavioral aspects through cognitive behavioral therapy, mindfulness training, and antidepressant medication, as well as education in sleep hygiene and exercise and diet regimens, can improve resilience [2]. Resilience-promoting programs that include strategies to improve sleep health, through modulation of sleep habits, have hitherto been lacking.

Resilience is known to be related to good sleep quality [3-5]. Poor sleep may negatively impact resilience and contribute to the development of mental health problems in children and adolescents $[3,6]$. Morningness is also known to be related to better re- 
silience $[3,6]$, whereas eveningness is associated with depression $[7,8]$. Morning-type children have reported less daytime sleepiness, while morning-type adolescents have fewer sleep-wake problems and morning-type female adolescents have reported fewer nocturnal symptoms [9]. Eveningness has been associated with attention deficit hyperactivity disorder [10-12], substance abuse [13], and suicide ideation [14].

Lee et al. [4] observed that morningness was related to several sleep-related modifiable habits, such as longer sleep duration, less social jetlag, and greater mean daily sunlight exposure between 10:00 and 15:00. Regarding sleep duration, there have been conflicting results; some studies found that eveningness was associated with short sleep duration [15], whereas some found no relation $[16,17]$. Regarding social jetlag, there was another study which found that less social jetlag was associated with morningness $[17,18]$. Regarding daytime sunlight exposure, morning-type adolescents have exhibited longer sunlight exposure [19]. Lee et al. [4] suggested that encouraging morning-type sleep habits may promote individuals' resilience. The importance of promoting healthy sleep habits has been demonstrated in relation to American military services [20]. If sleep-wake problems increase future psychological, physical, and occupational problems, encouraging healthy sleep habits may promote resilience, which protects individuals from future and present stressors.

Previous resilience-promoting programs have focused on social and emotional aspects [21]. The Penn Resiliency Program is aimed at strengthening children's and adolescents' mental capacity and increase their life satisfaction by promoting resilience [22]. However, this program mainly uses cognitive-behavioral approaches without managing sleep problems. Several other mental health promotion programs prioritize the development of coping skills, mindfulness, empathic relations, self-awareness, and help-seeking [21]. These programs have been effective in increasing resilience, but some were not effective in reducing depression and/or anxiety $[21,22]$. Therefore, we hypothesized that developing resilience-promoting programs that incorporate sleep behavior management in addition to cognitive-behavioral approaches could promote individuals' resilience.

In addition to resilience, smartphone addiction is problematic among adolescents and children. Smartphone addiction is known to be related to sleep quality and duration, and to chronotype [2325]. Eveningness in adolescents is known to be associated with higher levels of smartphone addiction [23]. Sleep quality has been reported to mediate the effects of smartphone addiction on adolescents' physical health [24], and shorter sleep duration has been associated with smartphone addiction in Korean children [25]. In young adults, social jet lag and smartphone use before bedtime have been associated with smartphone addiction in the context of eveningness [26]. Less exposure to sunlight is also correlated with smartphone addiction [26].

The authors aimed to develop a resilience-promoting program that can easily be delivered by staff members at mental health centers in Korea and that incorporates the encouragement of healthy sleep habits; the teaching of cognitive reappraisal approaches to stressful situations is also to be emphasized. We hypothesized that the program would be effective in improving resilience among adolescents. We also hypothesized that the program may help to alleviate addiction problems, including smartphone addiction, and that sleep-related factor(s) would predict resilience.

\section{METHODS}

\section{Program development}

Our resilience-promoting program was based mainly on the results of an earlier study [4] that demonstrated correlations of resilience with morningness and sleep quality, as well as with age, depression, and anxiety [4]. Good sleep quality was predicted by prolonged sunlight exposure between 10:00 and 15:00 [4]. Morningness was associated with lower social jetlag, longer total sleep time, and longer sunlight exposure between 10:00 and 15:00 [4]. Based on these findings, we aimed to develop a community resilience promotion program applicable to all age groups [27]. We aimed to incorporate the concept of cognitive reappraisal into the program. Several example stressful situations were devised, applicable to a wide age range, along with exercises requiring cognitive reappraisal in response to disappointing circumstances.

\section{Participants}

To test the program's effectiveness, we contacted staff at a mental health-promotion center in Haman-gun, who agreed to implement the program among the community in Haman-gun $\left(128^{\circ}\right.$ $25^{\prime} \mathrm{E} / 35^{\circ} 15^{\prime} \mathrm{N}$ ). Haman-gun is located in the center of Gyeongsangnamdo and has four distinct seasons. Five staff at a mental healthcenter in Haman-gun were trained to conduct the program, as stated above. The training lasted 30 minutes; the staff were instructed to read proscribed information to the participants regarding the program, without adding any personal comments. Fifty-eight middle school students (27 female, 46.6\%; aged 13.78 \pm 0.82 years) were recruited to the study. The staff conducted the program in four middle schools in Haman-gun in the morning before the start of classes. Four weekly sessions were conducted with the students between March and July 2017. During each 40-minute session, one staff member worked with three to four students. During the first and final sessions, several self-reported questionnaires, including the Connor-Davidson Resilience Scale (CD-RISC) [28], the Smartphone Addiction Scale-short version (SAS-SV) [29], Hospital Anxiety and Depression Scale (HADS) [30], Composite Scale of Morningness (CSM) [31], Insomnia Severity Index (ISI) [32], and a sleep habit-related questionnaire were administered to the students. After the first session, students were provided with an Actiwatch (Philips/Respironics, Murrysville, PA, USA) and were asked to wear the watch to the last session date for three weeks.

\section{Ethics statement}

Written informed consent was obtained from all participants, 
and at least one of their parents. Before conducting the program at the school, the mental health center staff provided detailed information about the program to the participants, as stated above, and asked them to carefully consider whether they wished to participate. The Institutional Review Board of Gyeongsang National University Hospital approved the study protocol (IRB file number: 2015-06-004-004).

\section{Self-reported questionnaires}

The CD-RISC is a simple self-report tool for measuring resilience that was devised by Connor and Davidson [28]. The scale comprises 25 questions, each scored on a 5-point scale ranging from 0 to 4 . The CD-RISC is used to evaluate various aspects of resilience in clinical and general populations [28]. Total scores range from 0 to 100, and high scores reflect high resilience [28]. The CD-RISC has shown high validity and reliability for measuring resilience in Korean populations [1,33]. The internal reliability in this study was good (Cronbach's a for CD-RISC $=0.89$ and 0.90 in the first and fourth sessions, respectively). The SAS$\mathrm{SV}$ is a shortened version of the SAS that was developed to evaluate the tendency toward, rather than diagnose smartphone addiction. The original version consists of 33 questions, and the shortened version comprises 10 questions [29]. Each item is scored from 1 point to 6 points, and a high score indicates a high risk of smartphone addiction. According to the scale's developer, the cutoff score for a high risk of smartphone addiction is 31 for men and 33 for women. The Korean version of SAS-SV has high validity and reliability [29]. The internal reliability in this study was good (Cronbach's $\alpha=0.89$ and 0.84 in the first and fourth sessions, respectively). The HADS is a self-report tool designed to screen for anxiety and depression in outpatients. It consists of anxiety and depression subscales (HADS-A and HADS-D, respectively; 14 items in total) [30]. Each question is scored on a 3-point basis starting from 0 , and each subscale has a cutoff of 8 points. The Korean version has also shown good validity and reliability [34]. The internal reliability in this study was adequate (Cronbach's $\alpha=0.78$ and 0.65 for HADS-A and HADS-D, respectively, for the first session; and 0.85 and 0.84 , respectively, for the fourth session).

The CSM is a self-report questionnaire developed by Smith et al. [31] to identify chronotypes. The scale consists of 13 questions; 3 of these are scored from 1 to 5 points, and the remaining 10 are scored from 1 to 4 points [31]. The total scores thus range from 13 to 55 [31]. Higher scores indicate a greater likelihood of morningness [31]. For the Korean version, a score of 41 or higher indicates morning type, 27 to 40 indicates neither type, and 26 of lower denotes evening type [35]. Good reliability and validity have also been confirmed for the Korean version [35]. The internal reliability in this study was good (Cronbach's $\alpha=0.83$ and 0.82 for the first and fourth sessions, respectively). The ISI is a self-report measure designed to assess the quality of sleep [32]. It consists of seven questions, with each question scored from 0 to 5 points [32]. The total score is between 0 and 28 , and a higher score reflects more severe insomnia [32]. The internal reliability in this study was acceptable (Cronbach's $\alpha=0.82$ and 0.76 for the first and fourth sessions, respectively). Sleep habits were also evaluated. Social jetlag, which refers to the difference between social time and endogenous time, was calculated based on the absolute value of the difference in awakening time between weekdays and weekends. Sunlight exposure time was considered as the outdoor activity time between 10:00 and 15:00 on weekdays and weekends, including activities such as walking, exercising, and sitting on a bench.

\section{Actigraphy data}

Actigraphy data were collected using the Actiwatch Spectrum (Philips/Respironics). This device measures wrist movement time series using the digital integration method. The data were downloaded using Actiware software (version 3.4; Philips/Respironics) and imported to Actiware 5 (versions 5.57 and 5.59, Respironics) for analysis. Spectrum devices can also detect whether the device is on or off the wrist; here off-wrist periods were designated as missing. Wake after sleep onset (WASO) is the total minutes of wakefulness between the initiation and termination of the sleep period and does not include sleep onset latency.

\section{Statistical analysis}

The CD-RISC and CSM scores for the first and fourth sessions, HADS-D scores for the first session, SAS-SV scores for the fourth session, sleep efficiency during the first week, WASO for the first and fourth sessions, and awakening frequency during the first and third weeks were normally distributed, as assessed by the Shapiro-Wilk test. HADS-A and ISI scores for the first and fourth sessions, HADS-D scores for the fourth session, SAS-SV scores for the first session, mean sunlight exposure between 10:00 and 15:00 and social jet lag for the first and fourth sessions, sleep efficiency during the third week, and sleep onset latency during the first and third weeks were not normally distributed. The nonparametric variables before and after the program were compared using the related-samples Wilcoxon signed-rank test. Parametric data that met the criteria for the paired $t$-test were analyzed using that test. Multiple linear regression analyses were used to identify predictors of resilience, smartphone addiction, sleep quality, and morningness/eveningness among the variables that showed significant differences after the program. All analyses were performed using SPSS for Windows (ver. 23.0; IBM Corp., Armonk, NY, USA). A two-tailed $\mathrm{p}$-value $<0.05$ was considered to indicate statistical significance.

\section{RESULTS}

A resilience-promoting program consisting of four 40-minute weekly sessions was developed. Each session could be completed individually or in small groups (up to five persons). The first session includes an introduction to the program and explains the concept of mental health and resilience and the importance of promoting resilience. In the first session, participants are asked to assess their own social jetlag based on the absolute value of the 
difference between their weekday and weekend rise times. In this session, participants are educated as to how they can reduce social jetlag to within 30 minutes. They also calculate their subjective sleep efficiency. The second session includes brief guidance with respect to applying cognitive reappraisal to stressful situations. Several cases are presented, and one verbatim session is introduced as an example. The third session includes education about healthy sleep with general information about how to acquire healthy sleep and advice regarding the reduction of social jetlag while suggesting longer sleep times and longer sunlight exposure between 10:00 and 15:00. The fourth session is a wrap-up session, which includes a re-assessment of participants' depression, anxiety, resilience, morningness/eveningness, quality of sleep, social jetlag, and sleep efficiency. They are encouraged to evaluate their own changes regarding these values and to apply the program's content continually to their daily lives. During the first to third sessions, participants were asked to complete a daily sleep diary and wear the actigraphy device as "homework"; 37 (63.79\%) participants complied with these requirements. The contents of each session are presented in Table 1.

The participants' resilience, morningness/eveningness, sleep quality, WASO, and awakening frequency during night changed significantly (Table 2). However, anxiety, depression, mean sunlight exposure time, sleep onset latency, and sleep efficiency (actigraphy) did not change significantly (Table 2). The SAS-SV score significantly improved (Table 2 ). In the first session, 10 (17.24\%) adolescents were classified as evening type, $42(72.41 \%)$ as neither type, and $6(10.34 \%)$ as morning type. In the fourth session, 5 (8.62\%) adolescents were classified as evening type, 46 (79.31\%) as neither type, and $7(12.07 \%)$ as morning type. In the first session, $19(32.76 \%)$ participants tended toward smartphone addiction; this number decreased to $16(27.59 \%)$ at the fourth session.

To identify predictors of resilience among the factors that were improved after the resilience-promoting program had been delivered, we performed multiple linear regression analysis (Table 3). Resilience at the fourth session was related to sleep quality at the fourth session when controlling for age, sex, and morningness/ eveningness (Table 3). To identify predictors of SAS-SV, we performed another multiple linear regression analysis (Table 4). SASSV was related to the mean sunlight exposure time at the fourth session when controlling for age, sex, depression, and sleep quality at the fourth session, and the mean sunlight exposure time at the first session (Table 4). Another multiple linear regression analysis was conducted to identify the predictors of sleep quality at the fourth session (Table 5). The mean sunlight exposure time at the fourth session was significantly related to sleep quality at the fourth session when controlling for age, sex, and WASO during the third week (Table 5). To identify the predictors of morningness/eveningness, we conducted a multiple linear regression analysis (Table 6). Morningness/eveningness at the fourth session was significantly related to the mean sunlight exposure time at the fourth session when controlling for age, sex, and WASO during the third week (Table 6).

\section{DISCUSSION}

Four 40-minute sessions of the resilience-promoting program, conducted at the mental health center, improved the participants' resilience. This is the first study to report improved resilience through modifying sleep-related behaviors. The tendency toward smartphone addiction was also improved after the program. Compared to the first session, sleep quality was improved, and the proportion of participants showing morningness increased.

Our resilience-promoting program is easy to implement, and is focused mainly on a cognitive-behavioral intervention for modifying sleep-related habits and educating individuals about cognitive reappraisal techniques that can be applied to stressful situations. After completing the program, participants' tendency toward smartphone addiction was decreased, which was not the primary outcome initially desired when the program was being developed. However, depression and anxiety showed no change after the program. Previous programs targeting adolescents' resilience have

Table 1. Contents of the resilience-promoting program by session

\begin{tabular}{ll}
\multicolumn{1}{c}{ Session } & \multicolumn{1}{c}{ Contents } \\
First session (40 min) & - Introduction to the concept of resilience \\
& - Self-rating of resilience, anxiety, depression, and insomnia severity \\
& - Calculation of social jetlag and sleep efficiency \\
& - Homework: wear actigraphy device, complete sleep diary \\
Second session (40 min) & - Example stressful situations \\
& - Education on cognitive reappraisal in response to stressful situations through case studies \\
& - Homework: wear actigraphy device, complete sleep diary \\
Third session (40 min) & - Healthy sleep instructions \\
& - Advice on how to reduce social jetlag, and achieve a longer sleep duration and more sunlight exposure between \\
& 10:00 and 15:00 \\
& - Homework: wear actigraphy device, complete sleep diary \\
Fourth session (40 min) & "Wrap-up" \\
& - Re-check of levels of resilience, anxiety, depression, and insomnia severity \\
& - Calculation of changes in social jetlag and sleep efficiency
\end{tabular}


Table 2. Baseline demographic data of participants and the comparison of scores on self-reported questionnaires and actigraphic data at first and fourth sessions of the resilience-promoting program $(n=58)$

\begin{tabular}{|c|c|c|c|c|c|}
\hline & First session & Fourth session & $\begin{array}{l}\text { Correlation } \\
\text { coefficient }(\mathrm{r})\end{array}$ & $\mathrm{W}^{*} / \mathrm{t}^{\dagger}$ & p-value \\
\hline Age (yr) & $13.78 \pm 0.82$ & - & & & \\
\hline Female & $27(46.6)$ & - & & & \\
\hline CD-RISC score & $65.08 \pm 12.33$ & $68.90 \pm 12.54$ & 0.44 & $1,131.50^{*}$ & 0.001 \\
\hline HADS-A score & $4.62 \pm 2.97$ & $4.47 \pm 3.49$ & -0.14 & $383.0^{*}$ & 0.272 \\
\hline HADS-D score & $4.26 \pm 2.74$ & $4.36 \pm 3.63$ & -0.04 & $488.50^{*}$ & 0.740 \\
\hline ISI score & $6.21 \pm 4.78$ & $4.41 \pm 3.57$ & -0.46 & $206.50^{*}$ & $<0.001$ \\
\hline CSM score & $32.22 \pm 6.31$ & $34.38 \pm 6.14$ & 0.52 & $1,158.50^{*}$ & $<0.001$ \\
\hline SAS-SV score & $30.66 \pm 10.50$ & $26.43 \pm 8.40$ & -0.46 & $292.50^{*}$ & 0.001 \\
\hline $\begin{array}{l}\text { Mean sunlight exposure between } \\
\text { 10:00 and 15:00 (hr) }\end{array}$ & $1.41 \pm 0.84$ & $1.46 \pm 0.97$ & 0.05 & $624.0^{*}$ & 0.712 \\
\hline Social jetlag (hr) & $2.28 \pm 1.48$ & $2.02 \pm 1.29$ & -0.18 & $478.5^{*}$ & 0.181 \\
\hline Sleep efficiency (\%) & $85.42 \pm 4.16(\mathrm{n}=46)$ & $85.91 \pm 5.13(n=37)$ & 0.09 & $348.0^{*}$ & 0.589 \\
\hline Sleep onset latency (min) & $19.27 \pm 13.94(n=46)$ & $17.70 \pm 14.16(n=37)$ & 0.20 & $242.0^{*}$ & 0.232 \\
\hline WASO (min) & $41.10 \pm 14.96(n=46)$ & $35.01 \pm 13.22(\mathrm{n}=37)$ & -0.40 & $2.573^{\dagger}$ & 0.015 \\
\hline Awakening frequency (no) & $45.56 \pm 13.24(\mathrm{n}=46)$ & $40.05 \pm 12.71(n=37)$ & -0.38 & $179.0^{*}$ & 0.026 \\
\hline
\end{tabular}

Data are expressed as $\mathrm{n}(\%)$ or mean \pm standard deviation. ${ }^{*}$ related samples Wilcoxon signed-rank test; ${ }^{\dagger}$ paired t-test. CD-RISC, Connor-Davidson Resilience Scale; HADS-A, Hospital Anxiety and Depression Scale-Anxiety; HADS-D, Hospital Anxiety and Depression Scale-Depression; ISI, Insomnia Severity Scale; CSM, Composite Scale of Morningness; SAS-SV, Smartphone Addiction Scale-short version; WASO, wake after sleep onset

Table 3. Multiple linear regression model for resilience measured by CD-RISC $(n=58)$

\begin{tabular}{lcccc} 
& $\mathrm{B}$ & Standard error & $\beta$ & \multicolumn{2}{c}{$\mathrm{p}$-value } \\
Intercept & 32.770 & 29.583 & 0.273 & 0.167 \\
Age $(\mathrm{yr})$ & 2.557 & 1.920 & 0.045 & 0.189 \\
Sex (male=1, female=0) & 1.118 & 3.093 & -0.394 & 0.005 \\
ISI score at fourth session & -1.383 & 0.469 & 0.091 & 0.504 \\
CSM score at fourth session & 0.186 & 0.277 & \\
\hline
\end{tabular}

Dependent variable: $\mathrm{CD}$-RISC at fourth session. $\mathrm{R}^{2}=0.20$, adjusted $\mathrm{R}^{2}=0.14 . \mathrm{F}=3.37$ ( $\mathrm{p}=0.016$ ). CD-RISC, Connor-Davidson Resilience Scale; ISI, Insomnia Severity Scale; CSM, Composite Scale of Morningness

Table 4. Multiple linear regression model for smartphone addiction measured by SAS-SV $(n=58)$

\begin{tabular}{lrrrr} 
& B & Standard error & $\beta$ & p-value \\
Intercept & 14.230 & 17.222 & 0.412 \\
Age (yr) & 1.078 & 1.225 & 0.105 & 0.383 \\
Sex (male=1, female= 0) & 0.189 & 1.972 & 0.011 & 0.924 \\
HADS-D score for fourth session & 0.349 & 0.339 & 0.151 & 0.308 \\
ISI score for fourth session & 0.256 & 0.350 & 0.109 \\
Mean sunlight exposure during 10:00 to 15:00 for the fourth session & -3.703 & 1.057 & -0.428 & 0.468 \\
\hline
\end{tabular}

Dependent variable: SAS-SV for the fourth session. $\mathrm{R}^{2}=0.30$, adjusted $\mathrm{R}^{2}=0.24$. $\mathrm{F}=4.52$ ( $\left.\mathrm{p}=0.002\right)$. SAS-SV, Smartphone Addiction Scale-short version; HADS-D, Hospital Anxiety and Depression Scale-Depression; ISI, Insomnia Severity Scale

incorporated social and emotional learning, mindfulness, stress management, or emotional wellbeing [21]. Recent research has explored the bi-directional relationship between sleep and resilience in adolescence [36], so we tested the efficacy of a sleep intervention to improve resilience. Our program focused primarily on changes in sleep habits. To our knowledge, our recommendations to reduce social jet lag, increase sleep duration, and prolong sunlight exposure between 10:00 and 15:00 as means of improving resilience constitute the first attempt at this approach. Sleep quality also improved as a result of the program, and this was the relevant factor predicting resultant resilience. At the fourth session, sleep quality was associated with duration of sunlight exposure between 10:00 and 15:00. Previous research has shown that sunlight exposure between 10:00 and 15:00 is associated with better resilience in young adults [4]. Among the encouraged sleep habits, the lengthening of sunlight exposure between 10:00 and 15:00 was found to be the only predictor for better sleep quality, and shorter sunlight exposure was reported to be a predictor for poor sleep [37,38]. Sunlight is an important zeitgeber for endogenous rhythms, and sunlight exposure may have a crucial influence on 
Table 5. Multiple linear regression model for sleep quality measured by ISI $(n=37)$

\begin{tabular}{lcccc} 
& B & Standard error & $\beta$ & \\
Intercept & -3.105 & 9.620 & 0.749 & 0.429 \\
Age $(\mathrm{yr})$ & 0.565 & 0.705 & 0.130 & -0.132 \\
Sex (male=1, female=0) & -0.797 & 1.008 & -0.435 \\
Mean sunlight exposure during 10:00 to 15:00 for the fourth session & -0.990 & 0.455 & 0.037 \\
WASO for the fourth session & 0.046 & 0.038 & 0.204 \\
\hline
\end{tabular}

Dependent variable: ISI for the fourth session. $\mathrm{R}^{2}=0.21$, adjusted $\mathrm{R}^{2}=0.11$. $\mathrm{F}=2.09$ ( $\left.\mathrm{p}=0.105\right)$. ISI, Insomnia Severity Scale; WASO, wake after sleep onset

Table 6. Multiple linear regression model for morningness/eveningness as measured by the CSM ( $\mathrm{n}=37)$

\begin{tabular}{lrrrr} 
& B & Standard error & $\beta$ & p-value \\
Intercept & 50.779 & 17.985 & 0.008 & -0.181 \\
Age (yr) & -1.503 & 1.319 & -263 \\
Sex (male=1, female=0) & -1.053 & 1.884 & -0.092 & 0.580 \\
Mean sunlight exposure during 10:00 to 15:00 for the fourth session & 2.035 & 0.851 & 0.391 & 0.023 \\
WASO for the fourth session & 0.069 & 0.071 & 0.159 \\
\hline
\end{tabular}

Dependent variable: CSM for the fourth session. $\mathrm{R}^{2}=0.24$, adjusted $\mathrm{R}^{2}=0.14$. $\mathrm{F}=2.49$ ( $\left.\mathrm{p}=0.063\right)$. CSM, Composite Scale of Morningness; WASO, wake after sleep onset

human sleep and mental health through vitamin $\mathrm{D}$ photosynthesis and ultraviolet A (315-400 nm)-induced release of NO, which act as a neurotransmitter in the blood circulation [39]. Further studies are needed to clarify the relationship between sunlight and sleep quality.

This program, which was aimed at improving resilience, also reduced adolescents' tendency toward smartphone addiction. Psychological interventions adopting cognitive-behavioral approaches are known to be effective for reducing smartphone addiction among adolescents [40]. Group intervention and educational programs were also effective in reducing the severity of Internet/smartphone addiction [40]. Our resilience-promoting program reduced social jetlag and promoted morning-type sleep habit and sunlight exposure, thereby significantly reducing adolescents' tendency toward smartphone addiction. The best predictor of smartphone addiction was mean sunlight exposure between 10:00 and 15:00. However, sleep quality and morningness were not found to be significant predictors of adolescent smartphone addiction. The mechanism by which sunlight exposure predicts the tendency toward smartphone addiction was not determined. Ultraviolet light exposure may have an addictive element [41], so might satisfy supplant smartphone use in individuals showing a tendency toward additive behaviors. However, this is the first attempt to improve smartphone addiction by modifying sleep-related factors such as social jetlag, daytime sunlight exposure, and sleep efficiency. The mechanism by which lower sunlight exposure promotes smartphone addiction is unknown, and longitudinal studies will be needed to further explore this.

Following four weekly sessions of the resilience-promoting program, participants showed significant improvement in sleep quality, as measured by the ISI. As none of our participants suffered from insomnia, whether the findings are applicable to adolescents with insomnia remains unclear. In addition to subjective reports, actigraphic measures-WASO and awakening frequency during sleep-were significantly decreased. Previous meta-analysis has revealed that cognitive-behavioral therapy for insomnia in children and adolescents improved sleep onset latency and sleep efficiency but not WASO and total sleep time [42]. A previous groupdelivered cognitive behavioral therapy for insomnia showed no significant improvement in actigraphic WASO in adolescents [43]. Data regarding awakening frequency measures after intervention in adolescents were lacking. As actigraphic WASO and awakening frequency during sleep are sleep quality measures, these results showed improved objective sleep quality measures after the resilience-promoting program. The role of sleep quality in resilience should be further assessed in larger studies using a longitudinal cohort design.

A shift toward morningness was observed after the resiliencepromoting program. Eveningness usually peaks during late adolescence, and a shift toward eveningness is observed during puberty [44]. A shift toward morningness after sleep intervention has been observed in adult populations [45], and such shifts have been associated with improved sleep quality and increases in positive affect [45]. As morningness/eveningness was changeable in response to sleep intervention focusing on behavioral change, the authors suggested that morningness/eveningness could exhibit state-like constructs along with trait-like aspects [45]. Our adolescent participants also showed significant changes toward morningness, and their morningness was associated with daytime sunlight exposure duration, which was one of the sleep-related practices encouraged by the resilience-promoting program. Previous studies have reported that sunlight exposure in the morning along with nutritional support promoted morningness in young children [46,47]. Morning-type adolescents had longer daytime sunlight exposure [19]. Although the duration of daytime sunlight exposure did not change significantly after our program, morningness/eveningness at the fourth session was significantly predicted by sunlight exposure duration at the fourth session. The 
cross-sectional nature of the analysis impeded our drawing robust conclusions regarding the morningness-promoting effects of the sleep-related practices promoted by the program.

Enhanced resilience after the program was significantly associated with sleep quality at the fourth session. Good sleep may promote resilience, because neural plasticity is enhanced during deep sleep [3]. Neural plasticity in resilient individuals functions as a protective factor against developing psychopathology when experiencing new environments [3]. Morningness was not related to resilience in the final session of this study, although previous cross-sectional studies of healthy adults have shown that morningness is associated with better resilience $[4,48]$. To verify the relationship between morningness and resilience in adolescents, future large-scale longitudinal studies will be necessary.

This study had several limitations that should be addressed. First, as there was no follow-up, the program's long-term effects could not be evaluated. Second, the sample size was too small to detect small to medium effect sizes. In multiple regression analysis, a sample size of 53 to 55 can detect medium to large effect sizes [49]. Third, the participants were middle school students, and sunlight exposure was not possible during school hours. Fourth, the subjective nature of self-report measures should be acknowledged when interpreting the results. Fifth, participants' degree of commitment to the program may have influenced the results. Among 58 participants, 37 (63.8\%) wore the actigraphy device for 3 weeks, which was one of the program requirements. This compliance rate suggests that their commitment to the program was good. Sixth, as the results of multiple linear regression analysis were based on cross-sectional data, no cause-and-effect assumptions could be drawn. Seventh, because of the homogeneity of the study population, it is not appropriate to generalize the findings to other populations. Eighth, although the study followed a prospective cohort study design, no control group was included. Future longitudinal studies incorporating control groups and other age groups are recommended.

The study had several strengths. First, the authors used a prospective design to assess the effectiveness of the resilience-promoting program. Second, the developed program represented the first attempt to improve resilience through the modification of sleep habits. Third, the program, aimed primarily at promoting resilience, also improved the adolescents' smartphone addiction.

In conclusion, we found that four weekly sessions of the resilience-promoting program improved resilience, smartphone addiction, sleep quality, including objective actigraphic measures, and morningness. Improved resilience was related to better sleep quality. Smartphone addiction was related to shorter daytime sunlight exposure. The adolescents' sleep quality and morningness were related to longer daytime sunlight exposure times.

\section{Acknowledgments}

This study was supported by a grant from the Korean Mental Health Technology R\&D Project, Ministry of Health \& Welfare, Republic of Korea (HM15C1108).

\section{Conflicts of Interest}

The authors have no potential conflicts of interest to disclose.

\section{Author Contributions}

Conceptualization: Hyoseung Kang, So-Jin Lee, Bong-Jo Kim. Data curation: Hyoseung Kang, So-Jin Lee, Jae-Won Choi, Young-Ji Lee, Yoon Jung Lee, Eun-Ji Lim. Formal analysis: Hyoseung Kang, SoJin Lee, Bong-Jo Kim, Cheol-Soon Lee, Boseok Cha, Dongyun Lee. Funding acquisition: Hyoseung Kang, So-Jin Lee. Investigation: Hyoseung Kang, So-Jin Lee, Dongyun Lee, Jiyeong Seo, JaeWon Choi, Young-Ji Lee, Yoon Jung Lee, Eun-Ji Lim. Methodology: So-Jin Lee, Bong-Jo Kim, Cheol-Soon Lee, Boseok Cha. Project administration: Hyoseung Kang, So-Jin Lee, Bong-Jo Kim, CheolSoon Lee, Boseok Cha, Jiyeong Seo. Resources: Dongyun Lee, Jiyeong Seo, Jae-Won Choi, Young-Ji Lee, Yoon Jung Lee, Eun-Ji Lim. Software: Dongyun Lee, Jiyeong Seo, Jae-Won Choi, Young-Ji Lee, Yoon Jung Lee, Eun-Ji Lim. Supervision: So-Jin Lee, Bong-Jo Kim, Cheol-Soon Lee, Boseok Cha, Dongyun Lee. Validation: So-Jin Lee, Bong-Jo Kim, Cheol-Soon Lee, Boseok Cha, Dongyun Lee. Visualization: Dongyun Lee, Jiyeong Seo, Jae-Won Choi, Young-Ji Lee, Yoon Jung Lee, Eun-Ji Lim. Writing_original draft: Hyoseung Kang, So-Jin Lee, Young-Ji Lee, Yoon Jung Lee. Writing-review \& editing: Hyoseung Kang, So-Jin Lee, Bong-Jo Kim, Cheol-Soon Lee, Boseok Cha, Dongyun Lee.

\section{ORCID iDs}

Hyoseung Kang (1)

https://orcid.org/0000-0002-6852-4316

So-Jin Lee (10

https://orcid.org/0000-0003-2904-9206

Bong-Jo Kim (c)

https://orcid.org/0000-0003-2419-7306

Cheol-Soon Lee (1)

https://orcid.org/0000-0003-1479-6962

Boseok Cha (1)

https://orcid.org/0000-0002-3309-8863

Dongyun Lee (1)

https://orcid.org/0000-0002-3977-3663

Jiyeong Seo (1)

https://orcid.org/0000-0002-7329-8296

Jae-Won Choi (1)

https://orcid.org/0000-0002-4516-1954

Young-Ji Lee (10)

https://orcid.org/0000-0003-0201-2518

Yoon Jung Lee (1)

https://orcid.org/0000-0003-4208-7039

Eun-Ji Lim (1)

https://orcid.org/0000-0003-3967-8524

\section{REFERENCES}

1. Baek HS, Lee KU, Joo EJ, Lee MY, Choi KS. Reliability and validity of the Korean version of the Connor-Davidson Resilience Scale. Psychiatry Investig 2010;7:109-115. 
2. Southwick SM, Charney DS. The science of resilience: implications for the prevention and treatment of depression. Science 2012;338:79-82.

3. Chatburn A, Coussens S, Kohler MJ. Resiliency as a mediator of the impact of sleep on child and adolescent behavior. Nat Sci Sleep 2013;6:1-9.

4. Lee SJ, Park CS, Kim BJ, Lee CS, Cha B, Lee YJ, et al. Association between morningness and resilience in Korean college students. Chronobiol Int 2016; 33:1391-1399.

5. Puterman E, Epel ES, Lin J, Blackburn EH, Gross JJ, Whooley MA, et al. Multisystem resiliency moderates the major depression-telomere length association: findings from the Heart and Soul Study. Brain Behav Immun 2013; 33:65-73.

6. Leahy E, Gradisar M. Dismantling the bidirectional relationship between paediatric sleep and anxiety. Clin Psychol 2012;16:44-56.

7. Natale V, Adan A, Scapellato P. Are seasonality of mood and eveningness closely associated? Psychiatry Res 2005;136:51-60.

8. Gaspar-Barba E, Calati R, Cruz-Fuentes CS, Ontiveros-Uribe MP, Natale V, De Ronchi D, et al. Depressive symptomatology is influenced by chronotypes. J Affect Disord 2009;119:100-106.

9. Gelbmann G, Kuhn-Natriashvili S, Pazhedath TJ, Ardeljan M, Wöber C, Wöber-Bingöl C. Morningness: protective factor for sleep-related and emotional problems in childhood and adolescence? Chronobiol Int 2012;29: 898-910.

10. Imeraj L, Sonuga-Barke E, Antrop I, Roeyers H, Wiersema R, Bal S, et al. Altered circadian profiles in attention-deficit/hyperactivity disorder: an integrative review and theoretical framework for future studies. Neurosci Biobehav Rev 2012;36:1897-1919.

11. Caci H, Bouchez J, Baylé FJ. Inattentive symptoms of ADHD are related to evening orientation. J Atten Disord 20091;13:36-41.

12. Voinescu BI, Szentagotai A, David D. Sleep disturbance, circadian preference and symptoms of adult attention deficit hyperactivity disorder (ADHD). J Neural Transm (Vienna) 2012;119:1195-1204.

13. Urbán R, Magyaródi T, Rigó A. Morningness-eveningness, chronotypes and health-impairing behaviors in adolescents. Chronobiol Int 2011;28:238-247.

14. Selvi Y, Aydin A, Boysan M, Atli A, Agargun MY, Besiroglu L. Associations between chronotype, sleep quality, suicidality, and depressive symptoms in patients with major depression and healthy controls. Chronobiol Int 2010; 27:1813-1828

15. Soehner AM, Kennedy KS, Monk TH. Circadian preference and sleep-wake regularity: associations with self-report sleep parameters in daytime-working adults. Chronobiol Int 2011;28:802-809.

16. Robilliard DL, Archer SN, Arendt J, Lockley SW, Hack LM, English J, et al. The 3111 clock gene polymorphism is not associated with sleep and circadian rhythmicity in phenotypically characterized human subjects. J Sleep Res 2002;11:305-312.

17. Randler C, Jankowski KS. Evidence for the validity of the composite scale of morningness based on students from Germany and Poland-relationship with sleep-wake and social schedules. Biol Rhythm Res 2014;45:653-659.

18. Randler C. Proactive people are morning people. J Appl Soc Psychol 2009; 39:2787-2797.

19. Harada T, Morisane H, Takeuchi H. Effect of daytime light conditions on sleep habits and morningness-eveningness preference of Japanese students aged 12-15 years. Psychiatry Clin Neurosci 2002;56:225-226.

20. Peterson C, Park N, Castro CA. Assessment for the U.S. Army Comprehensive Soldier Fitness program: the global assessment tool. Am Psychol 2011; 66:10-18.

21. Fenwick-Smith A, Dahlberg EE, Thompson SC. Systematic review of resilience-enhancing, universal, primary school-based mental health promotion programs. BMC Psychol 2018;6:30.

22. Bastounis A, Callaghan P, Banerjee A, Michail M. The effectiveness of the Penn Resiliency Programme (PRP) and its adapted versions in reducing depression and anxiety and improving explanatory style: a systematic review and meta-analysis. J Adolesc 2016;52:37-48.

23. Randler C, Wolfgang L, Matt K, Demirhan E, Horzum MB, Beşoluk Ş. Smartphone addiction proneness in relation to sleep and morningness-eveningness in German adolescents. J Behav Addict 2016;5:465-473.

24. Xie X, Dong Y, Wang J. Sleep quality as a mediator of problematic smartphone use and clinical health symptoms. J Behav Addict 2018;7:466-472.

25. Kim SY, Han S, Park EJ, Yoo HJ, Park D, Suh S, et al. The relationship between smartphone overuse and sleep in younger children: a prospective cohort study. J Clin Sleep Med 2020;16:1133-1139.

26. Kim Y, Lee SJ, Park CS, Kim BJ, Lee CS, Cha B, et al. The mediating effect of eveningness on the indirect relationships between shorter sleep duration, inattention, depression with smartphone addiction tendency. Chronobiol Med 2020;2:32-40.

27. Kang H. Development of a resilience promotion program using changes in sleep-related behaviors in adolescents [dissertation]. Jinju: Gyeongsang National University; 2021.

28. Connor KM, Davidson JR. Development of a new resilience scale: the Connor-Davidson Resilience Scale (CD-RISC). Depress Anxiety 2003;18:76-82.

29. Kwon M, Lee JY, Won WY, Park JW, Min JA, Hahn C, et al. Development and validation of a smartphone addiction scale (SAS). PLoS One 2013;8:e56936.

30. Zigmond AS, Snaith RP. The hospital anxiety and depression scale. Acta Psychiatr Scand 1983;67:361-370.

31. Smith CS, Reilly C, Midkiff K. Evaluation of three circadian rhythm questionnaires with suggestions for an improved measure of morningness. J Appl Psychol 1989;74:728-738.

32. Cho YW, Song ML, Morin CM. Validation of a Korean version of the Insomnia Severity Index. J Clin Neurol 2014;10:210-215.

33. Jung YE, Min JA, Shin AY, Han SY, Lee KU, Kim TS, et al. The Korean version of the Connor-Davidson Resilience Scale: an extended validation. Stress Health 2012;28:319-326.

34. Oh SM, Min KJ, Park DB. A study on the standardization of the Hospital Anxiety and Depression Scale for Koreans: a comparison of normal, depressed and anxious groups. J Korean Neuropsychiatr Assoc 1999;38:289296.

35. Yoon JS, Shin SM, Kook SH, Lee HY. A preliminary study on the Korean translation of Composite Scale (KtCS) to measure morningness-eveningness. J Korean Neuropsychiatr Assoc 1997;36:122-134.

36. Wang J, Zhang X, Simons SR, Sun J, Shao D, Cao F. Exploring the bi-directional relationship between sleep and resilience in adolescence. Sleep Med 2020;73:63-69.

37. Leger D, Bayon V, Elbaz M, Philip P, Choudat D. Underexposure to light at work and its association to insomnia and sleepiness: a cross-sectional study of 13,296 workers of one transportation company. J Psychosom Res 2011;70: 29-36.

38. Marqueze EC, Vasconcelos S, Garefelt J, Skene DJ, Moreno CR, Lowden A. Natural light exposure, sleep and depression among day workers and shiftworkers at arctic and equatorial latitudes. PLoS One 2015;10:e0122078.

39. Juzeniene A, Brekke P, Dahlback A, Andersson-Engels S, Reichrath J, Moan K, et al. Solar radiation and human health. Rep Prog Phys 2011;74:066701.

40. Malinauskas R, Malinauskiene V. A meta-analysis of psychological interventions for Internet/smartphone addiction among adolescents. J Behav Addict 2019;8:613-624.

41. Keen SB, Yelverton CB, Rapp SR, Feldman SR. UV light abuse as a substancerelated disorder: clinical implications. Arch Dermatol 2008;144:1047-1048.

42. Ma ZR, Shi LJ, Deng MH. Efficacy of cognitive behavioral therapy in children and adolescents with insomnia: a systematic review and meta-analysis. Braz J Med Biol Res 2018;51:e7070.

43. de Bruin EJ, Oort FJ, Bögels SM, Meijer AM. Efficacy of internet and groupadministered cognitive behavioral therapy for insomnia in adolescents: a pilot study. Behav Sleep Med 2014;12:235-254.

44. Jankowski KS. Composite Scale of Morningness: psychometric properties, validity with Munich ChronoType Questionnaire and age/sex differences in Poland. Eur Psychiatry 2015;30:166-171.

45. Hasler BP, Buysse DJ, Germain A. Shifts toward morningness during behavioral sleep interventions are associated with improvements in depression, positive affect, and sleep quality. Behav Sleep Med 2016;14:624-635.

46. Nakade M, Takeuchi H, Taniwaki N, Noji T, Harada T. An integrated effect of protein intake at breakfast and morning exposure to sunlight on the circadian typology in Japanese infants aged 2-6 years. J Physiol Anthropol 2009;28:239-245.

47. Nakade M, Akimitsu O, Wada K, Krejci M, Noji T, Taniwaki N, et al. Can breakfast tryptophan and vitamin B6 intake and morning exposure to sunlight promote morning-typology in young children aged 2 to 6 years? J Physiol Anthropol 2012;31:11.

48. Antúnez JM, Navarro JF, Adan A. Circadian typology is related to resilience and optimism in healthy adults. Chronobiol Int 2015;32:524-530.

49. Cohen J. A power primer. Psychol Bull 1992;112:155-159. 